\title{
Interior Path Planning Method Based On Main Road
}

\author{
FengBin ${ }^{1, a}$, XiaQian, ${ }^{2, b}$, Carlos A. Acosta ${ }^{3, c}$ \\ ${ }^{1}$ Guangzhou Institute of Geography, China \\ ${ }^{2}$ Guangzhou Bo Jin Information Technology Co., Ltd., China \\ ag23548055@qq.com, b13260572654@163.com
}

\begin{abstract}
Keywords: main road, indoor navigation, path planning, algorithm
Abstract. With the rapid development of social economies, the complexity of buildings and their equally elaborate inner environments are on the rise-causing indoor navigation problems to be gradually placed on the agenda. In this paper, integrating a method of interior path planning based on the "main road" concept is proposed - which is distinct from the traditional algorithm based on a* and $\mathrm{d}^{*}$ algorithm. Based on this "main road" idea, the advance compilation of indoor environment plans and the incorporation of the user's common path as the "main road" are stored creating a more concise path planning. The location of users, the destinations and key points in the relation to the "main road" are selected to determine the best route for the user. Simultaneously, the starting position on the main road is determined from the point of origin. The system does not need to calculate the next path. The system only needs to plan the nearest location to the main road and generate an auxiliary path and road map for the main road. Experimental results show that the proposed method saves a lot of node computation and path planning time, and provides a more efficient and convenient method for indoor path planning.
\end{abstract}

\section{Introduction}

In our daily life, the satellite positioning navigation system is very good to meet the needs of our outdoor activities. With the development of social economy and the progress of urban building technology a greater number of larger buildings are introduced into people's lives. These buildings are not only tall and magnificent but their inner environments are also complex and diverse. When people are in a building such as an airport, a high-speed rail station, an underground car park or a government service center, (even when there is a site map and logo in such buildings), due to large number of floors, wide areas, the complex internal environment as well as an increasing number of other factors such as human flow, etc., the system cannot often locate the "road". This is compounded even more when the information has not been updated, thus often times causing the system to appear as either continually searching or "jumping" from mock locations. These situations are not only a waste of people's time but also make it physically and mentally exhausting-greatly impacting the user's experience. At this time and age, the indoor navigation system has become increasingly critical. The core of indoor navigation systems is embodied in the path-planning algorithm, the advantages and disadvantages of the algorithm determine the effectiveness of the room navigation.

Scholars at home and abroad have conducted research around the path planning - part of which revolves around the path planning of the mobile robot, and the other part incorporates the A* algorithm, a $\mathrm{D}^{*}$ algorithm or $\mathrm{A} *$ algorithm, a $\mathrm{D}^{*}$ algorithm for path planning. We have briefly incorporated the findings from three of papers here within our paper in an effort to demonstrate the strengths and weaknesses of their proposed methods in comparison to ours. The first document identified as [1] describes the individual indoor navigation algorithm based on Mimu. Their proposed use is specialized and unsuitable for the general public's daily use. In the second paper marked as [2], the construction of indoor road network is realized by triangulation. However, it requires large amounts of artificial interaction, causing long algorithm response time and low path planning efficiency. Finally, in the third paper labeled as [3] proposes a method to construct the indoor pedestrian network for landmarks. However, the "things" that can be categorized as "landmarks" in the room are not invariable, thus this method can only be used in a small range. We have based our 
work and strategy in accordance with the research on the current situation at home and abroad. This paper puts forward the interior path planning algorithm based on the "main road" concept, which is distinct and apart from the traditional algorithm based on a* and $\mathrm{d}^{*}$ algorithm, and obtains the main path of the indoor environment by preprocessing. In fact, the part that needs to be calculated is the optimal path of matching the ideal main road as well as the starting point of the main road. Test trials conducted show that the proposed method can meet the needs of indoor path planning.

\section{The interior path planning method based on the main road}

In this paper, the indoor Path planning method is based on the main road using the indoor plan, in accordance with the user's own needs by quickly extracting the path required by the user. The main road with the "expressway" promptly extracts the optimal path between the starting position and the endpoint. With this method, users can quickly and accurately access the "Freeway" design that thinks in terms of a specific environment based on the needs of the users providing rapid extraction and design of the common main road and the appropriate main road for the user's navigation and path search. This method can quickly and accurately extract the optimal path for the user, and reduce the time and resource consumption of real-time computation.

The main steps of this method are as follows:

(i) Analysis of indoor site characteristics, extraction of floor plans, key points of information, and storage;

(ii) Analysis of user needs, obtain the path of user traffic, extract common path information, and classify storage;

(iii) Merging the characteristics of the site, extracting the optimal path of each user's demand as the main road from the common path information, and combining the floor plan to generate the main road map and encode the storage;

(iv) Combine user location and destination information, from the main road map to extract the actual requirements for the main road map to complete the path planning in accordance with the actual needs of users.

Step (i) includes the preparatory work on the indoor location, extraction of the indoor floor plan with key points, and execution of most efficient and appropriate backup storage.

Step (ii) encompasses the analysis of the user's needs, obtaining the location information of the user, determining the starting and end point of the path in accordance with the utilizer's destination information, selecting the key points in the road according to the user's choice of route based on the starting point, the key point and the end point, and planning the common path and encoding

Step (iii) is primarily based on the user's needs. It incorporates the origin of the starting and end point as well as key points, according to the shortest path and minimum principle from the common path to choose the optimal path as the main road. At the same time, according to the actual needs of users, it combines the user location and destination information from the main road map to extract the actual required trunk road map.

Step (iv) extracts the optimized main road map from the main road map according to the user destination information. Based on the location information of the user as it corresponds to the principle of the shortest path and the minimum support, the ideal path map is extracted from the optimized main road map as the necessary main road map to complete the path planning. The main road map is also included according to the location information of the user, combined with the actual required trunk diagram. The optimal auxiliary road map of the main road map is generated according to the principle of the most accessible and shortest path. In the main or auxiliary road maps, the link elements are displayed by annotation, each corresponding to different types of key points, respectively, using different colors, symbols and text for display. In the Main and Auxiliary road maps, the dynamic icon displays the user's real-time location information as well as showing the user's location in regards to the distance of the destination. 


\section{Interior path planning algorithm based on the Main road}

Basic Overview. We all know that the superhighway can make us more efficient and expeditious in reaching our destination, the main road concept can also be analogous to the "superhighway." The interior path planning algorithm based on the main road can quickly extract the optimal path between the starting and the endpoint. This unconventional Interior path planning (node model, network triangulation) idea is completely from a single point (or network triangulation) - complexly calculated one by one to form a coherent thought, from which the small number of main roads would directly be extracted. This creates a new level of thought process in interior path planning - taking it to the next level.

Algorithm Steps. The method of interior path planning based on the main road is divided into the data preprocessing algorithm and the real-time processing algorithm. The preprocessing algorithm steps are detailed as follows, as shown in Figure 1:

S1: Analysis of indoor site characteristics, extraction of floor plans and key points of information and storage. The characteristics of indoor venues are typically categorized as: high-speed railway stations, airports, comprehensive government service centers and shopping malls. Due to the distribution of most functional areas, the entire coverage of a wider area, and a large flow of people, a high-speed, fast indoor navigation technology is very important for users. In most of these areas, the floor plans usually already exist — needing only for the key point information to be marked and the floor plan obtained. Key point adjustments include: exits, entrances, turning points, elevators, emergency exits, staircases, toilets or other functional areas specific to the actual site.

S2: Analysis of user needs, find the walking path for the user, extract common path information, and classify storage. This step includes the set up of the most commonly used path information in advance, its characteristics for the site which is inclusive of the following four steps as shown in Figure 2: S2-1: Obtain the location information of the user and determine the starting point of the path; S2-2: Determine the end path, according to the user's destination information; For example: the user needs to go to the waiting area, but is currently at the ticket office... A line would be set from the ticket office to the waiting area then await the user's choice of route. Once they select the initial route, the system would indicate the next decision-making process to determine the next steps the user must take. S2-3: The user's preferred Chosen Path would determine the key points in the path. Therefore, in order to enhance the user's experience and choice of implementation, the system needs to combine the most commonly used path and specific frequency of use, such as elevators, etc. Simultaneously upon choosing of a route, the key points in the path map are determined to provide users with a clearer and easier identifiable path planning experience; S2-4: Throughout the starting point, the key point and the end point, the common path is planned, as well as the encoding storage classification.

S3: Combining the site characteristics from the common path information, the optimal path corresponding to each user's demand is extracted from the main road and combined with the floor plan. The main road map is then generated and the encoded storage is divided into several steps, as follows: According to the user needs the starting, end, and key points are determined in conformance with the principle of maximum path and shortest route. The optimal path is then chosen as the main road from the common path information.

S4: According to the user's actual needs, the location and destination information accessed from the main road map will be integrated in order to extract the actual needs for the main road map to complete the path planning. This process would include the following steps, as shown in Figure 3: S4-1: Extract the optimal main road from the main road map according to the user's destination information. This is taken separately from the destination information. The optimized trunk diagram automatically extracts from hundreds of common path information of about 10-20 - which is much simpler than the existing complex computation. Higher efficiency is simultaneously achieved, as the consumption flow is very low. S4-2: The most unobstructed primary road as well as the shortest route based on the location information of the user is extracted to generate the optimal path map from the optimized trunk road map thus forming the actual necessary trunk diagram to complete the path 
planning. After step s4-1 there are more than 10 paths that lead to the selection of the most suitable main road map in accordance with the smoothest and shortest primary route, thus completing the path planning. To further enhance the outcome of their experience, when extracting the main road map, the distance information of the user's location as it pertains to the destination is also included and displayed. The goal is to visually display the distance the user needs to reach the destination and enable them to make an informed decision as to whether they chose to continue. For example, let's say the user wants to go to Starbucks to have a cup of coffee but they find that the distance from their present location is 1000 meters away. At this point, the user may choose not to go to Starbucks, and select instead to go to a nearby McDonald's or KFC, so on and so forth. In this practical application, the user's destination location (endpoint) is marked on the main road, hence creating a quicker and much more efficient way to plan the new route.

The real-time processing algorithm steps are as follows:

When users are inside a high-speed rail station or other large buildings that have an indoor navigation system based on this algorithm, all they would simply need to do is enter the destination information into the Path search box and choose the method of implementation such the most convenient route, the most accessible path, etc. Along this path of planning and control plans, users can quickly reach their destination. So, what kind of process is this path-planning algorithm? Combined with the flowchart shown in Figure 4, the following steps are implemented: (program)

- Obtain the user's current location information and set to the starting point;

- Get the destination information entered by the user and set the end point;

- The key points needing attention in the path planning of the query result based on the input destination are selected, and the optimal trunk path is selected.

Determine whether the user's current position is in the planned main road based on the starting point. If the user is on the main road path, the user can walk directly along the planned main Road. If the user is no longer on the trunk path, the algorithm generates a secondary path into the main road and generates a secondary road map into the trunk road map. The user goes along the planned route to the main road with the auxiliary road map and reaches the destination along the trunk path.

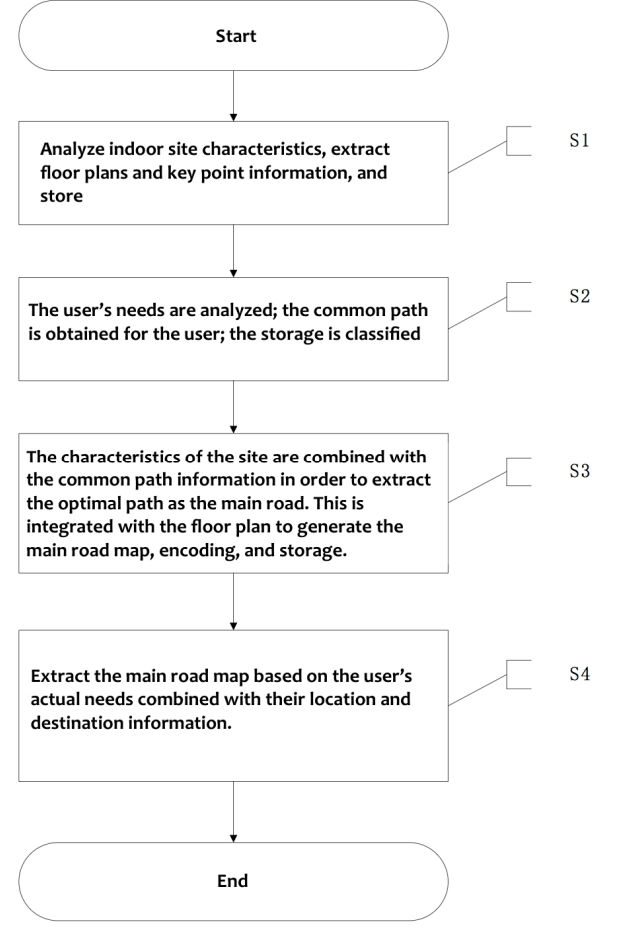

Figure 2 overall flowchart

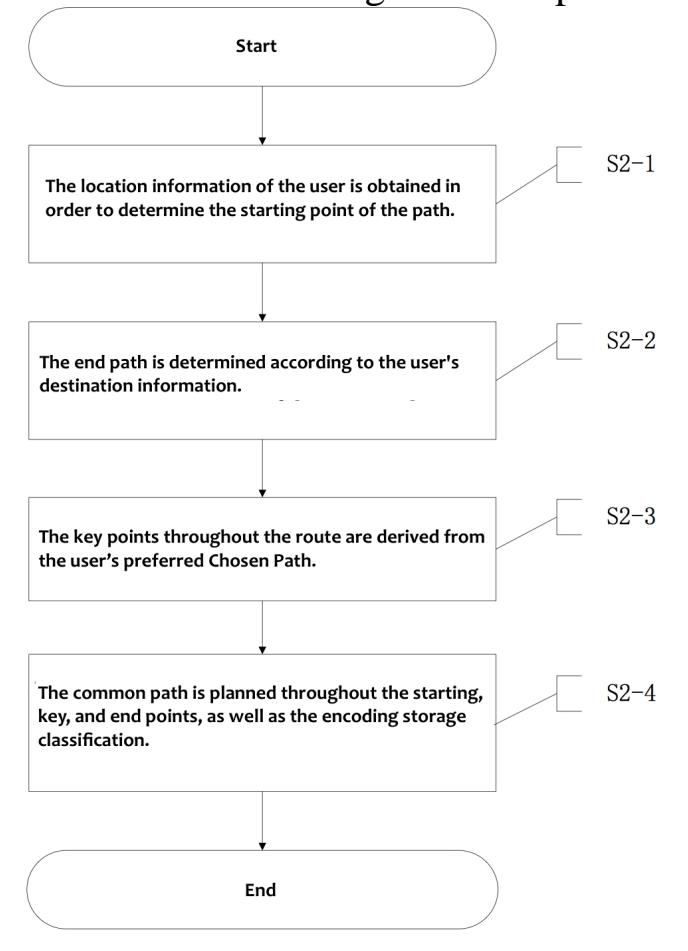

Figure 1 common path storage 


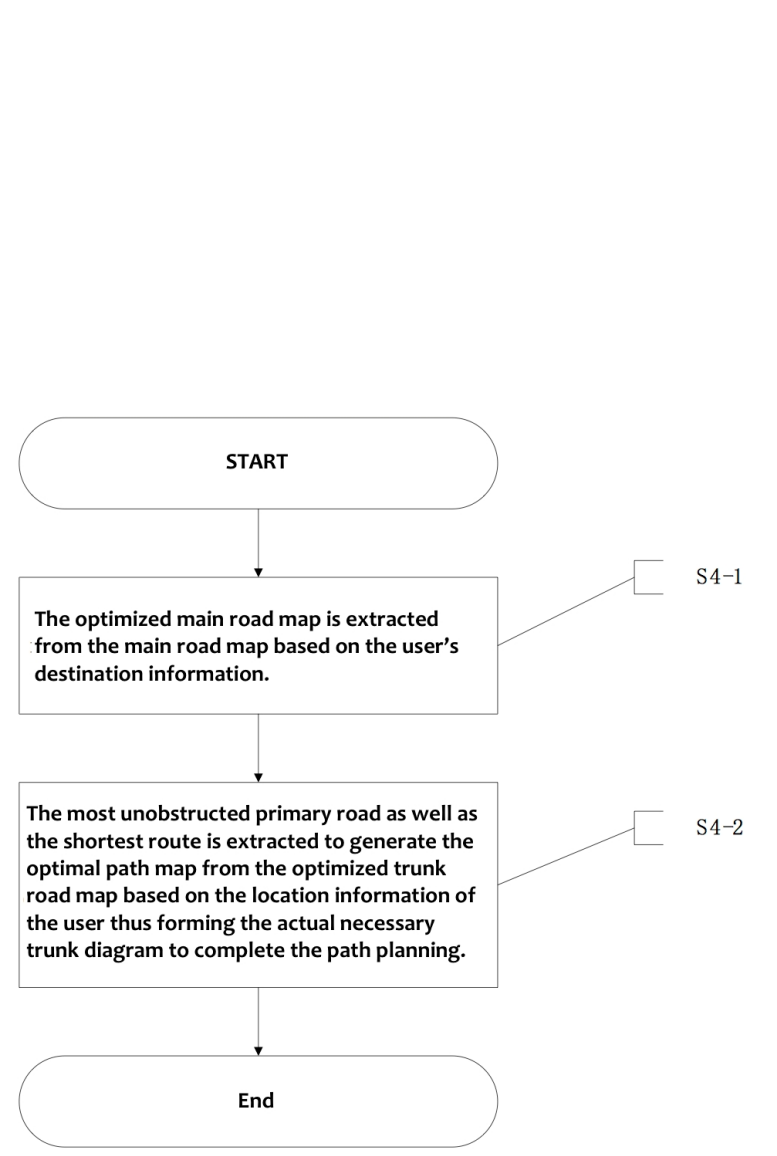

Figure 4 extract the actual required main road map

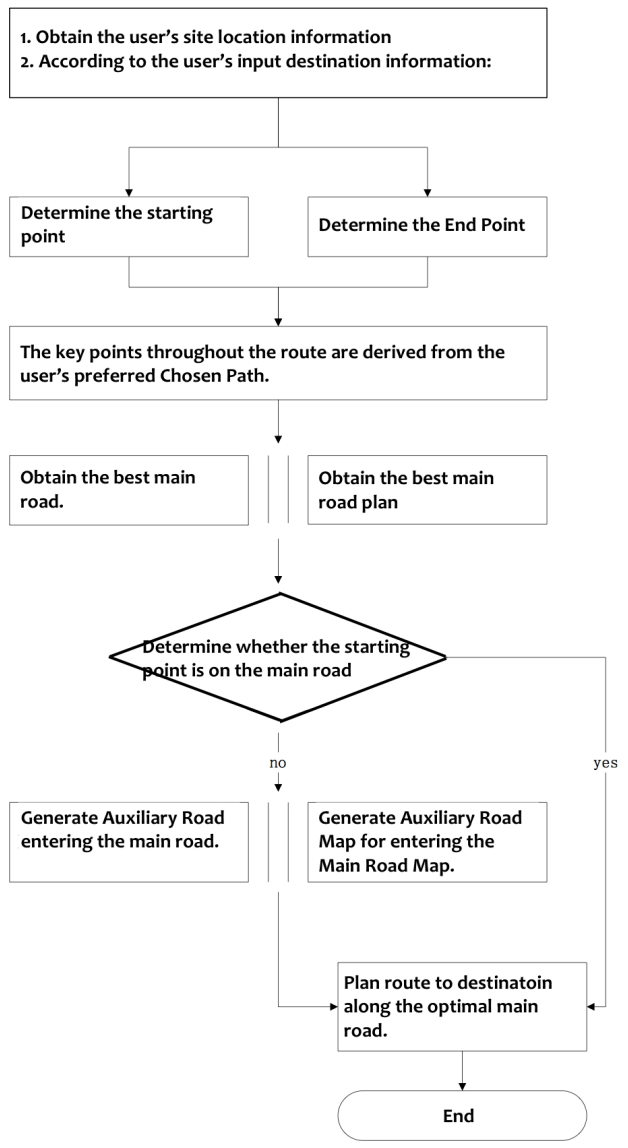

Figure 3 path planning process

\section{Analysis of the results from the test trials}

Background of experimental testing area. The trial site was the Guangzhou South Train Station with a total construction area of more than 370,000 square meters. The overall construction included the main station area, a no pillar rain shed, elevated yards (platforms), parking, and so on, with the total investment of 13 Billion Yuan. The main structure consisted of four levels, including three floors on the ground and one underground level - the underground floor is a subway to and from the station Hall, as well as parking area for 1,808 parking spaces. The ground level consists mainly of the passenger station operation for the Wu-Guang passenger train Line, the Guangzhou-Zhuhai intercity station, the Guang-Shen-Hong-Kong high speed Railway Train passenger's entry and exits, arrival hall, ticket offices, visitor's Joint Inspection Offices (immigration/customs), departure hall and the airport transport office. The two ground floors include the port entrance hall and the VIP lounge and so on, as well as the platform level, arranged from east to west facing the Wuhan down yard, the Guang-shen-Kong yard, the Wuhan up yard, the Guang Zhu Intercity, the Hiroshige, the main court yard, and the South court yard. The three ground level are equipped with dedicated escalators leading to the passenger waiting areas. The upper level waiting area measures 71,722 square meters, and includes a reserved area of 14,694 square meters of commercial floors.

Experimental Test Results. This paper focuses on the results of the Guangzhou South Railway station first floor test area (measuring 3600 square meters). Using the method described in this paper along with the node algorithm model to test the indoor navigation, the entire data for the site plan was used as the test area. The test consisted of four paths to the North Two car parking area destination: Guangzhou South station Exit B to North Two parking lot; Guangzhou South Station Exit D to North Two car parking area; Guangzhou South Station Exit F to North Two car parking area; and finally Guangzhou South Station Exit G to North Two car parking area.

The four sets of data navigation test paths were obtained as shown in Figure 5, and figure 6. 


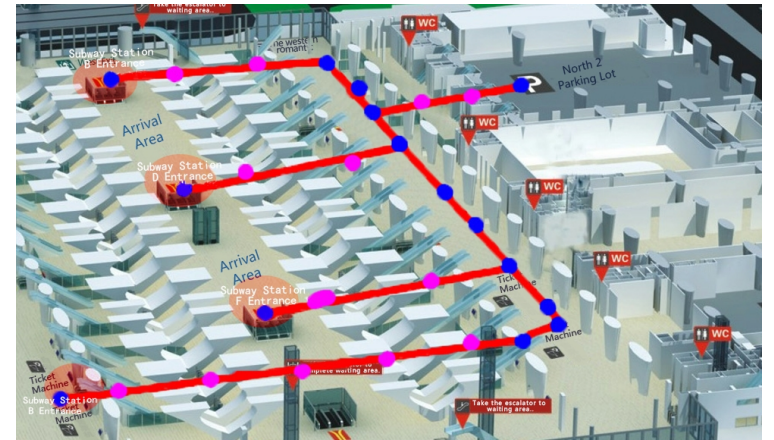

Figure 5 The planned path created by this method (Blue represents the key point, with Pink as the auxiliary point judged by the starting point and the end point)

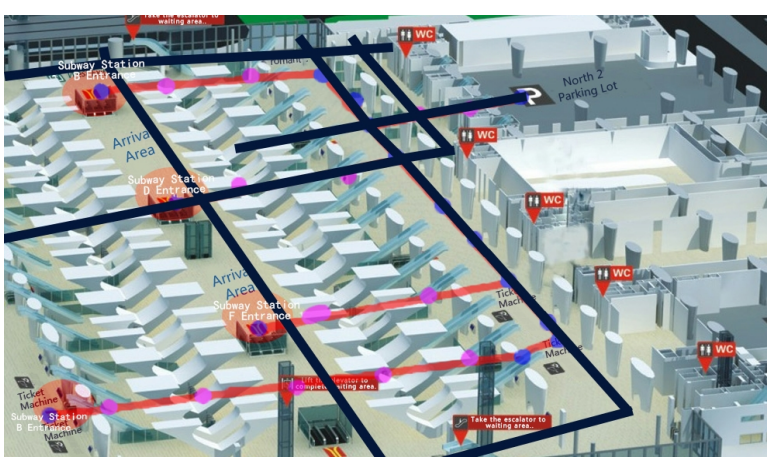

Figure 6 The path created by the node model planning (Example: the path planning for Metro exit D to the North Two car park)

By FIG. 5, the four paths that are planned by this method are simple and clear, and the starting point of each path is derived from the main road and planned in advance with a short response time.

As shown in Figure 6, the next step in planning is to employ the node model to design the path. Each adjustment will probe the location of the next node and repeat until the destination is finally reached.

\section{Experimental Analysis}

Using the method of indoor path planning based on main road, we have planned four paths, the total number of turning points is 21 , and the total number of key points is 35 . Compared with the path of using the node model and the algorithm planning based on triangular grid search, the time and the number of nodes obtained in this method are far less than the node model algorithm.

Table 1 is the statistical result of the method comparison between our method and the node model with the algorithm planning path based on triangular grid search. The main data includes the turning point (total), the key point (total), the turning point (single path), the key point (single path) and the planning time between five aspects. The analysis from the table shows that by comparison, it is found that the method proposed in this paper has fewer nodes than the other method, and that the time required for planning the path is better than that of the other method. Therefore, the method described in this paper can effectively solve the problem of indoor path planning, and the longer the path to be planned, the greater the advantages of the method expressed in this paper.

Table 1 statistical result

\begin{tabular}{|c|c|c|c|c|c|c|c|}
\hline Method & Path start & $\begin{array}{l}\text { Path } \\
\text { Endpoint }\end{array}$ & $\begin{array}{l}\text { Turning } \\
\text { Point } \\
\text { (total) }\end{array}$ & $\begin{array}{l}\text { Key } \\
\text { points } \\
\text { (total) }\end{array}$ & $\begin{array}{l}\text { Turning } \\
\text { point } \\
\text { (single } \\
\text { path) }\end{array}$ & $\begin{array}{l}\text { Key point } \\
\text { (single } \\
\text { path) }\end{array}$ & $\begin{array}{l}\text { Path } \\
\text { planning } \\
\text { time elapsed } \\
\text { between }\end{array}$ \\
\hline \multirow{4}{*}{$\begin{array}{l}\text { Node } \\
\text { model } \\
\text { algorithm }\end{array}$} & MTR station B exit & \multirow{4}{*}{$\begin{array}{l}\text { North } \\
\text { two } \\
\text { parking } \\
\text { lot }\end{array}$} & \multirow[t]{4}{*}{64} & \multirow[t]{4}{*}{44} & 16 & 12 & 0.8 \\
\hline & Subway Station D exit & & & & 16 & 12 & 0.8 \\
\hline & MTR Station F exit & & & & 16 & 12 & 0.8 \\
\hline & Subway Station G exit & & & & 16 & 12 & 0.8 \\
\hline \multirow{4}{*}{$\begin{array}{l}\text { This } \\
\text { algorithm }\end{array}$} & MTR station B exit & \multirow{4}{*}{$\begin{array}{l}\text { North } \\
\text { two } \\
\text { parking } \\
\text { lot }\end{array}$} & \multirow[t]{4}{*}{9} & \multirow[t]{4}{*}{15} & 3 & 5 & 0.06 \\
\hline & Subway Station D exit & & & & 2 & 4 & 0.05 \\
\hline & MTR Station F exit & & & & 2 & 7 & 0.07 \\
\hline & Subway Station G exit & & & & 4 & 10 & 0.08 \\
\hline
\end{tabular}

\section{Conclusions}

In this paper, the method of indoor path planning based on the main road is proposed. The goal of our method is to solve the existing problem of searching in large spaces as well as address the dilemma of long response time experienced by the traditional algorithm methods. This paper combines the 
thought of the main road, rationally plans the optimal main road and key point, and makes the main road as the target node, narrowing the computational space of the algorithm. The path of common trunk road is planned in advance, the calculation amount of node is reduced and the search efficiency is improved. The experiment and results prove the feasibility and effectiveness of the path-planning algorithm. At the same time, the interior path-planning method based on the main road concept facilitates the management of the relevant factors, with the priority being to ensure the patency of the main road, thus enabling users to navigate quickly to their destination with great ease.

\section{Acknowledgements}

This work was supported by the GDAS' Special Project of Science and Technology Development (2017GDASCX-0808).

\section{References}

[1] Ma Xiaorong. Research on the algorithm of individual soldier indoor navigation based on Mimu [D]. University of National Defense Science and Technology, 2013.

[2] Lou Wei, Wei, Zhangshuo, et. Research and implementation of construction method of indoor road network model [J]. Journal of Navigation and Positioning, 2014 (4): 63-67.

[3] Liu Tao, Xing, Li Qingquan, et. Interior navigation Path optimization algorithm considering landmark visibility [J]. Journal of Wuhan University (Information Science Edition), 2017, 42 (1): 43-48. 